\title{
CATATONIA AS A MANIFESTATION OF SYSTEMIC LUPUS ERYTHEMATOSUS: A CASE REPORT
}

Maria Luiza Rodrigues Barbosa Melo (Universidade Federal de Campina Grande, Campina Grande, PB, Brasil), Evânia Claudino Queiroga Figueiredo (Universidade Federal de Campina Grande, Campina Grande, PB, Brasil)

\section{BACKGROUND}

The neuropsychiatric manifestations of systemic lupus erythematosus (SLE) can precede the onset of the disease or can occur at any moment of its course. The pathogenesis of catatonia induced by SLE is not totally defined yet. However, it is thought to be caused by autoimmune lesion to the nervous central system and associated to antinuclear antibodies as the ribosomal anti-P. The use of corticosteroids can cause neuropsychiatric manifestations that are very similar to those caused by SLE. Giving that corticosteroids are widely used by patients with autoimmune diseases, it can be difficult to establish the cause of such neuropsychiatric manifestations.

\section{CASE REPORT}

An 18 year old female presented with intense skin paleness, malar rash, mouth ulcers, enlarged cervical lymph nodes, bilaterally symmetric arthritis in hands, ankles and knees and pancytopenia, as observed through a complete blood count. She also had a positive result for the antinuclear antibody test. The diagnostic of SLE was then established and treatment was then started, with oral hydroxychloroquin and prednisone. However, two weeks after that, the patient started having psychomotor agitation, insomnia, tremors, visual and auditory hallucinations and delirium, alternating with moments of waxy posture, difficulty walking and dysphagia to both solids and liquids. After admission to the hospital, our patient presented with catatonia and stopped making eye contact, was hypoactive, completely mute and responded very weakly to extern stimuli. She was also unable to feed due to severe trismus. Pulse steroid therapy was initiated, as well as treatment with benzodiazepines and and dopamine agonists. After a week of treatment the patient recovered well and was able to return home.

\section{CONCLUSION}

catatonia is one of the less frequent psychiatric manifestations of ESL, when compared to psychosis, for example. Its management can also be more difficult, due to the lack of understanding about its pathogeny. There is a wide range of therapeutic options that are listed in literature. This patient had a satisfactory response when association was made between the immunosuppressive treatment and specific treatment for the psychiatric manifestations. 